\title{
Bayesian Methods for Predicting the Shape of Chinese Yam in Terms of Key Diameters
}

\author{
Mitsunori Kayano, ${ }^{1}$ Koki Kyo, ${ }^{2}$ and Mitsuru Hachiya ${ }^{3}$ \\ ${ }^{1}$ Research Center for Global Agromedicine, Obihiro University of Agriculture and Veterinary Medicine, Inada-cho, \\ Obihiro 080-8555, Japan \\ ${ }^{2}$ Department of Human Sciences, Obihiro University of Agriculture and Veterinary Medicine, Inada-cho, Obihiro 080-8555, Japan \\ ${ }^{3}$ Institute of Agricultural Machinery, BRAIN, National Agricultural and Food Research Organization, 1-40-2, Nisshin-cho, \\ Kita, Saitama-shi 331-8537, Japan \\ Correspondence should be addressed to Mitsunori Kayano; kayano@obihiro.ac.jp
}

Received 19 January 2017; Revised 6 July 2017; Accepted 13 July 2017; Published 6 September 2017

Academic Editor: Pankaj B. Pathare

Copyright (C) 2017 Mitsunori Kayano et al. This is an open access article distributed under the Creative Commons Attribution License, which permits unrestricted use, distribution, and reproduction in any medium, provided the original work is properly cited.

\begin{abstract}
This paper proposes Bayesian methods for the shape estimation of Chinese yam (Dioscorea opposita) using a few key diameters of yam. Shape prediction of yam is applicable to determining optimal cutoff positions of a yam for producing seed yams. Our Bayesian method, which is a combination of Bayesian estimation model and predictive model, enables automatic, rapid, and lowcost processing of yam. After the construction of the proposed models using a sample data set in Japan, the models provide whole shape prediction of yam based on only a few key diameters. The Bayesian method performed well on the shape prediction in terms of minimizing the mean squared error between measured shape and the prediction. In particular, a multiple regression method with key diameters at two fixed positions attained the highest performance for shape prediction. We have developed automatic, rapid, and low-cost yam-processing machines based on the Bayesian estimation model and predictive model. Development of such shape prediction approaches, including our Bayesian method, can be a valuable aid in reducing the cost and time in food processing.
\end{abstract}

\section{Introduction}

Chinese yam (Dioscorea opposita) is one of the most exported crops from Japan. The value of yam exports reached 1.89 billion JPY in 2013 [1]. About $90 \%$ of the total yield of yam in Japan was produced in two prefectures, Hokkaido (45.8\%) and Aomori (44.0\%), in 2012 [2]. In both prefectures, mechanical cultivation is used for rapid expansion of production. However, seed yams (seed tubers of yams), which are uniformly cutoff yams (Figure 1), are manually produced and require the effort of 300 people.h/ha. In order to reduce the cost of production and improve the yield of yams, mechanization for producing seed yams is required.

The problem in the mechanization of seed yam production is how to determine the cutoff positions for each yam. It is expected that a yam be uniformly cut with a desired weight and without much loss. Therefore, under the assumption of equal density among yams, it is required that the shape of the yam be measured, since the weight of each seed yam can be calculated using the shape and the cutoff positions.

A straightforward way to measure the shape of a yam is to scan a yam using sensors. However, this includes three problems: (1) cost of the sensor, (2) speed of the process, and (3) accuracy of the scanning (e.g., trichomes of a yam can reduce the accuracy of the scanning). Another way is to use images of yams for shape determination. Such an approach has been widely used in fruit/crop grading, classification and removal before shipment [3-6]. Computational and statistical methodologies have been provided [7-16]. In the case of producing seed yams, the problem is much simpler than the general problem mentioned above for fruits and crops; we can assume a regular pattern of yams (see Figure 1) and do not have to strictly check yam damage, because the purpose here is to know the shape of yams quickly without the use of many devices (i.e., a low-cost way). 


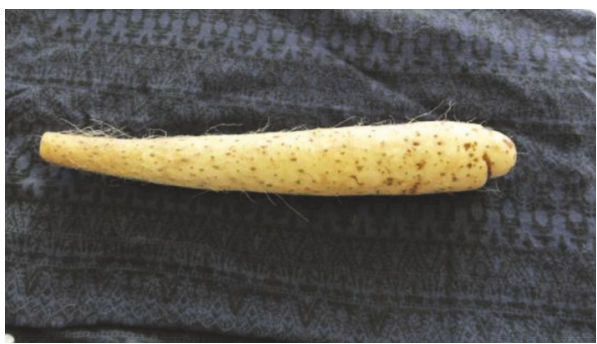

(a)

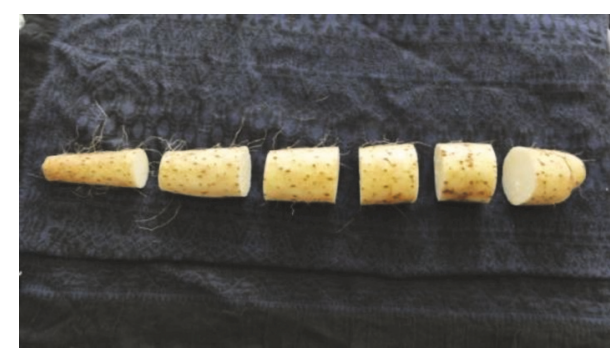

(b)

Figure 1: An example of yam (a) and seed yams (seed tubers of yam (b)).

In this paper, we propose a Bayesian framework to address issues (1) and (2), that is, to provide a low-cost and high-speed way for shape prediction of yam. Our hypothesis is that shape of yam can be predicted by a few key diameters at fixed positions, under an assumption that shape of yam can be represented by a set of diameters. In order to examine this hypothesis, we need to construct a model that gives a relationship between the diameters to be predicted and the key diameters, which can be measured. A difficulty in the model construction is that measurements of diameters for each sample are insufficient and unsteady. Thus, we introduce a Bayesian framework to relieve such difficulty.

Bayesian method is a technique for statistical inference that updates the probability based on a prior probability for random parameters in a model based on observations. By using Bayesian inference, we can set up a prior distribution for parameters based on prior information, which is available in advance, to obtain robust estimates for parameters for lack of observations, so Bayesian method is especially useful when observational data are insufficient for estimation. In this reason, methods of Bayesian data analysis are widely applied (e.g., [17]). Bayesian inference is particularly important in time series analysis. For example, [18] proposed an approach of Bayesian smoothness priors for analyzing time varying structure in a dynamic system; it is useful for a case that there are some missing data in time series. In this paper, we apply the technique of smoothness priors to the problem of shape prediction of Chinese yam.

The proposed method estimates the whole shape of a yam based on a few measurements of the key diameter of the yam. The two issues regarding the measurement of the shape of yams are overcome by using the proposed method, since the diameter of a yam are easily and accurately measured without any sensors. We estimated optimal positions of the diameter to be measured by minimizing the error of the shape prediction. We also illustrated high performance of the proposed method in terms of estimating the shape of yams using a sample data set, which contains the length, weight, and diameters at intervals of 10 to $50 \mathrm{~mm}$ (Figure 2, see also Section 2.2) of 111 yams from Hokkaido, Japan. After the construction of the proposed method using the sample data set, the method gives whole shape prediction of yam based on a few key diameters without any scanners or images of yam.

The rest of this paper is organized as follows; Section 2 discusses the procedures for implementing the proposed

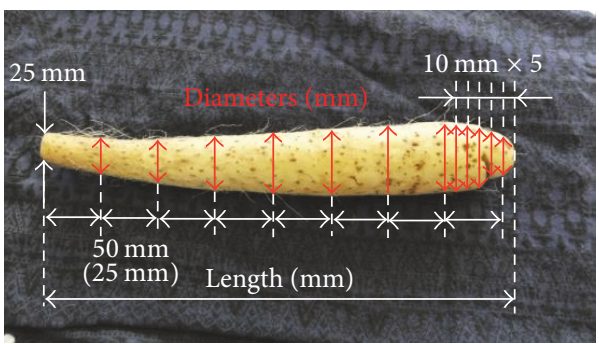

FIGURE 2: An example of the measurements of a yam: length and diameters. The weight was also observed. All yams were automatically cut off at a diameter of $25 \mathrm{~mm}$ (the cutoff point).

methods, the results obtained from a set of sample data are show in Section 3, and the result and performance of the proposed methods are discussed in Section 4. Finally, Section 5 concludes the paper.

\section{Materials and Methods}

2.1. Basic Consideration. In this section, we introduce our sample data set and proposed methods. After the construction of the proposed methods using the sample data set, the methods predict the whole shape of yam, which can be expressed by all the diameters along the length of a yam tuber shaft, based on a few key diameters that can be measured in advance.

We developed Bayesian methods to predict the shape of a yam in three steps.

Step 0. Arrange all yams into [0,1] interval (Figure 3).

Step 1. Apply Bayesian estimation model to estimate missing diameters (Figure 4).

Step 2. Construct Bayesian predictive model for shape prediction (Figure 5).

First of all, as Step 0 of our Bayesian methods, all yams are arranged into [0,1] interval (Figure 3). For example, in Figure $3,\left\{y_{i 6}, y_{i 12}, \ldots, y_{i 96}\right\}$ are actual observations, and $\left\{y_{i j}\right\}(j \neq 6,12, \ldots, 96)$, that is, $\left\{y_{i 1}, \ldots, y_{i 5}, y_{i 7}, \ldots\right\}$, are missing. We need a model to estimate all missing diameters. However, a problem is that the number of missing diameters 


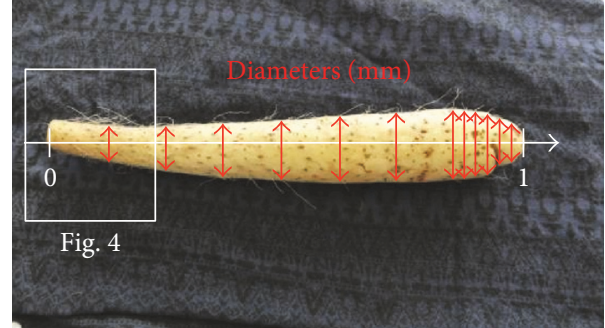

Figure 3: Step 0. All yams are arranged into $[0,1]$ interval.

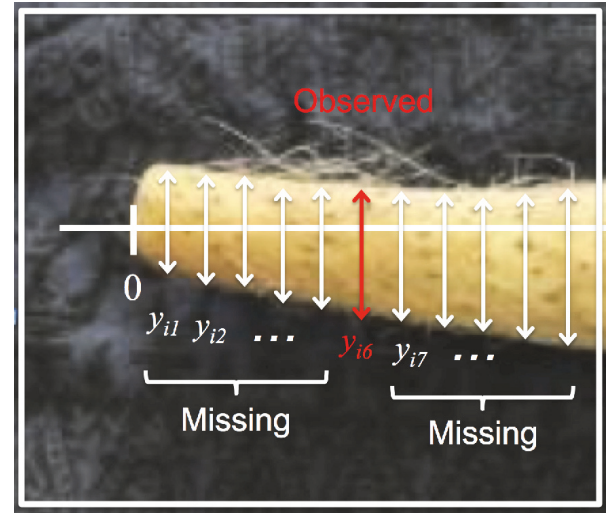

Figure 4: Step 1. An example of a set of missing diameters $\left(y_{i 1}, \ldots\right.$, $\left.y_{i 5}, y_{i 7}, \ldots\right)$ and observed diameters $\left(y_{i 6}, \ldots\right)$. All missing diameters are estimated by using Bayesian estimation model.

to be estimated exceeded that of the observations. Therefore, we applied the Bayesian model to solve this problem (Step 1). In Step 2, we constructed a predictive model based on the observed diameters and estimated diameters in Step 1. The details of the sample data set and proposed methods are explained in the following subsections.

2.2. Sample Data Set. In this study, we used data from $111(=M)$ yams in Hokkaido, Japan, to construct Bayesian models. Each yam had measurements of length ( $\mathrm{mm})$, weight (g), and diameters (mm) at suitable positions (Figure 2 and description below). All yams were automatically cut off at the position with a diameter of $25 \mathrm{~mm}$ (Figure 2). The mean length, weight, and diameter were $451.86( \pm 64.31) \mathrm{mm}$, $783.24( \pm 205.67) \mathrm{g}$, and $44.30( \pm 14.43) \mathrm{mm}$, respectively. The diameters were measured at intervals of $25 \mathrm{~mm}$ for 87 yams and $50 \mathrm{~mm}$ for 24 yams. Out of the 87 yams, 60 had detailed measurements of the diameter at intervals of $10 \mathrm{~mm}$ at the front edge of the yam. A scatterplot of the length and weight of the 111 yams in this study is shown in Appendix A. Length and weight were highly correlated with each other (Pearson correlation coefficient $r=0.739, p<0.001$ ), implying high quality of the data for model construction. Diameters. For a sample yam $i$, we consider the model for the

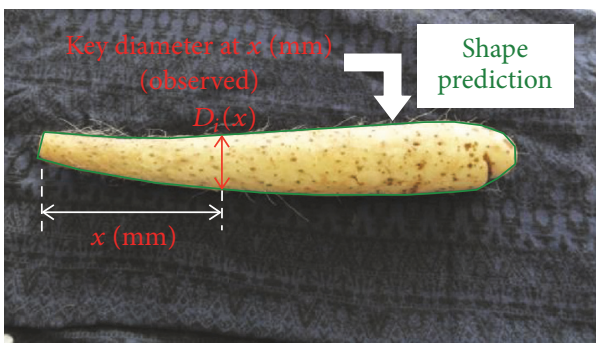

FIgURE 5: Step 2. Shape of a yam, that is, all diameters $\left\{y_{i j}\right\}(j=$ $1,2, \ldots, N)$ (both observed and estimated in Step 1), is predicted by a few key diameters $D_{i}(x)$ at $x(\mathrm{~mm})$. Mean squared error between the observed diameters and the predicted diameters is calculated in order to evaluate the prediction accuracy.

observation of the diameter at the $j$-th point as follows:

$$
y_{i j}=d_{i j}+\epsilon_{i j}, \quad(i=1,2, \ldots, M ; j=1,2, \ldots, N),
$$

where $y_{i j}, d_{i j}$, and $\epsilon_{i j}$ are the diameter, true diameter, and measurement error, respectively, $M$ is the number of yams in the sample, and $N$ is the number of equally spaced points for which the true diameter to be estimated. Note that when there is an observation near the $j$-th point, we regard it as the measure for $y_{i j}$; otherwise we consider that the $y_{i j}$ is missing.

A difficulty in estimating the unknown quantities $d_{i j}$ for $i=1,2, \ldots, M$ and $j=1,2, \ldots, N$ is that the number of the unknown quantities that need to be estimated is larger than that of the observations; that is, we have too many missing values for the diameters. In order to alleviate this difficulty, we used a Bayesian model. Here, from the viewpoint of a Bayesian approach, $d_{i j}$ is treated as a random variable. It is assumed that the distribution of this variable can be described with stochastic difference equations that are called smoothness priors ([18]). For a given sample $i$, we express the smoothness priors for $d_{i j}$ by a 2 -nd order stochastic difference equation as

$$
d_{i j}=2 d_{i(j-1)}-d_{i(j-2)}+v_{i j} \quad(j=1,2, \ldots, N) .
$$

In (1) and (2), $\epsilon_{i j} \sim N\left(0, \sigma^{2}\right)$ and $v_{i j} \sim N\left(0, \tau_{i}^{2}\right)$ are white noise sequences on $j$, and they are independent of each other, where $\sigma^{2}$ and $\tau_{i}^{2}$ are unknown parameters. By introducing the smoothness priors described in (2) into the model in (1), we can construct a set of flexible Bayesian linear models for $d_{i j}$.

Now, we put

$$
\begin{aligned}
\mathbf{z}_{i j} & =\left[\begin{array}{c}
d_{i j} \\
d_{i(j-1)}
\end{array}\right], \\
\mathbf{F} & =\left[\begin{array}{cc}
2 & -1 \\
1 & 0
\end{array}\right], \\
\mathbf{G} & =\left[\begin{array}{l}
1 \\
0
\end{array}\right], \\
\mathbf{H} & =\left[\begin{array}{l}
1 \\
0
\end{array}\right]^{\mathrm{t}} .
\end{aligned}
$$


Then, the model in (1) and (2) can be expressed by the following state space model:

$$
\begin{aligned}
& \mathbf{z}_{i j}=\mathbf{F} \mathbf{z}_{i(j-1)}+\mathbf{G} v_{i j}, \\
& y_{i j}=\mathbf{H} \mathbf{z}_{i j}+\epsilon_{i j} .
\end{aligned}
$$

In the state space model comprising (4), the parameter $d_{i j}$ is included in the state vector $\mathbf{z}_{i j}$, so its estimate can be obtained from the estimate of $\mathbf{z}_{i j}$. Moreover, the variances $\sigma^{2}$ and $\tau_{i}^{2}$ can be estimated by the maximum likelihood method. The above Bayesian model to estimate diameters of yams was first introduced in [19] for another application.

When the parameters $\sigma^{2}$ and $\tau_{i}^{2}$ are given, we can obtain the estimate of $\mathbf{z}_{i j}$ using the algorithm of Kalman filter. The estimates for parameters $\sigma^{2}$ and $\tau_{i}^{2}$ are obtained by maximizing a likelihood function which is defined based on the Kalman filter. See Appendix B for the algorithm of Kalman filter and Appendix C for the estimation of the parameters $\sigma^{2}$ and $\tau_{i}^{2}$ in detail. See also $[18,20]$.

2.4. Step 2: Bayesian Predictive Model for Shape Prediction Using Key Diameter(s). In this section, we propose three models for predicting the shape of a yam based on the results estimated from a set of samples. Let $D_{i}(x)$ be a key diameter at position $x(\mathrm{~mm})$ from the tip of the $i$ th yam (cf. Figure 5$)$. Also, let $D_{i}\left(x_{1}\right)$ and $D_{i}\left(x_{2}\right)$ be the key diameters at positions $x_{1}(\mathrm{~mm})$ and $x_{2}(\mathrm{~mm})$ from the tip of the $i$ th yam.

2.4.1. Weighted Averaging (WA). We aim to predict the diameters at all points $j=1,2, \ldots, N$ of a yam from the key diameters $D_{i}(x)$.

Defining $\widetilde{d}_{i j}(x)=\widehat{d}_{i j} / D_{i}(x)$ and $\widetilde{V}_{i j}(x)=V_{i j} / D_{i}^{2}(x)$, the posterior distribution of the normalized diameter $d_{i j} / D_{i}(x)$ is given by $N\left(\widetilde{d}_{i j}(x), \widetilde{V}_{i j}(x)\right)$, where $\widehat{d}_{i j}(x)$ is given by the first element of $\mathbf{z}_{i j \mid N}$, and $\widehat{V}_{i j}$ is given by the 1,1 element of $\mathrm{C}_{i j \mid N}$, which were obtained from the fixed-interval smoothing mentioned above. The weighted average of the diameters is then calculated by

$$
\begin{aligned}
\bar{d}_{j}(x) & =\sum_{i=1}^{M} w_{i j} \widetilde{d}_{i j}, \\
w_{i j} & =\frac{\widetilde{V}_{i j}^{-1}(x)}{\sum_{k=1}^{M} \widetilde{V}_{k j}^{-1}(x)}
\end{aligned}
$$

which can be regarded as the standard shape of the average yam.

Then, for a yam with the value $D^{*}(x)$ for the key diameter $D(x)$, its predicted diameter value at point $j$ is given by

$$
d_{j}^{*}(x)=\bar{d}_{j}(x) D^{*}(x) \text {. }
$$

\subsubsection{Regression Models (RM)}

Single Regression Model (S-RM). For the estimated value $\widehat{d}_{i j}$ of the diameter $d_{i j}$ and the value of key diameter $D_{i}(x)$, a single regression model is constructed as

$$
\begin{aligned}
\widehat{d}_{i j}= & a_{j}+b_{j} D_{i}(x)+e_{i j} \\
& e_{i j} \sim N\left(0, \psi_{i j}^{2}\right)(i=1,2, \ldots, M ; j=1,2, \ldots, N) .
\end{aligned}
$$

Then, we can obtain the estimates $\widehat{a}_{j}$ and $\widehat{b}_{j}$ of the regression coefficients $a_{j}$ and $b_{j}$ at point $j$ using a least squares method. For a given yam with a key diameter $D^{*}(x)$, the predictive value of the diameter at the point $j$ is obtained by $d_{j}^{*}(x)=$ $\widehat{a}_{j}+\widehat{b}_{j} D^{*}(x)$.

Multiple Regression Model (M-RM). Based on the estimated value $\widehat{d}_{i j}$ of the diameter $d_{i j}$ and the values of $D_{i}\left(x_{1}\right)$ and $D_{i}\left(x_{2}\right)$, a multiple regression model is built as

$$
\begin{aligned}
\widehat{d}_{i j} & =a_{j}+b_{j} D_{i}\left(x_{1}\right)+c_{j} D_{i}\left(x_{2}\right)+e_{i j}, \\
& e_{i j} \sim N\left(0, \psi_{i j}^{2}\right)(i=1,2, \ldots, M ; j=1,2, \ldots, N) .
\end{aligned}
$$

Then, the predictive value of the diameter at point $j$ is obtained using the relation $d_{j}^{*}=\widehat{a}_{j}+\widehat{b}_{j} D^{*}\left(x_{1}\right)+\widehat{c}_{j} D^{*}\left(x_{2}\right)$ with $\widehat{a}_{j}, \widehat{b}_{j}$, and $\widehat{c}_{j}$ being the estimates of the regression coefficients $a_{j}, b_{j}$, and $c_{j}$, respectively.

2.5. Evaluating the Performance of the Bayesian Methods. As mentioned above, three kinds of predictive models were constructed. There were two issues related to these predictive models. One was how to determine the location parameters, that is, $x$ in the WA and S-RM models or $x_{1}$ and $x_{2}$ in the M$\mathrm{RM}$ model. Another issue is how to evaluate these different models. A useful way to address these issues is the use of the mean squared error (MSE) as a criterion for evaluating the predictive models (see, e.g., [21]).

Specifically, for the WA and S-RM models, the MSE is defined by

$$
\operatorname{MSE}(x)=\frac{1}{N_{S}} \sum_{i=1}^{M} \sum_{j \in \mathrm{S}_{i}}\left\{y_{i j}-d_{i j}^{*}(x)\right\}^{2},
$$

where $d_{i j}^{*}(x)$ is the predictive value of the diameter at the $j$ th point on the $i$ th yam with the location parameter $x$, $S_{i}$ is the index set $\{1,2, \ldots, N\} \backslash R_{i}$ with the index set $R_{i}$ for missing values (so, $y_{i j}\left(j \in S_{i}\right)$ indicate the actual observations for $i$ th yam), and $N_{S}=\sum_{i=1}^{M}\left|S_{i}\right| \leq M N$ is the total number of indices with measurements. Thus, the mean square differences between predictive values and the observations for the diameters can be expressed. Therefore, we can determine the location parameter $x$ by minimizing the value of $\operatorname{MSE}(x)$ and then evaluate the predictive models based on the minimum values of $\operatorname{MSE}(x)$.

Similarly, for the M-RM model, MSE is defined by

$$
\operatorname{MSE}\left(x_{1}, x_{2}\right)=\frac{1}{N_{S}} \sum_{i=1}^{M} \sum_{j \in S_{i}}\left\{y_{i j}-d_{i j}^{*}\left(x_{1}, x_{2}\right)\right\}^{2},
$$




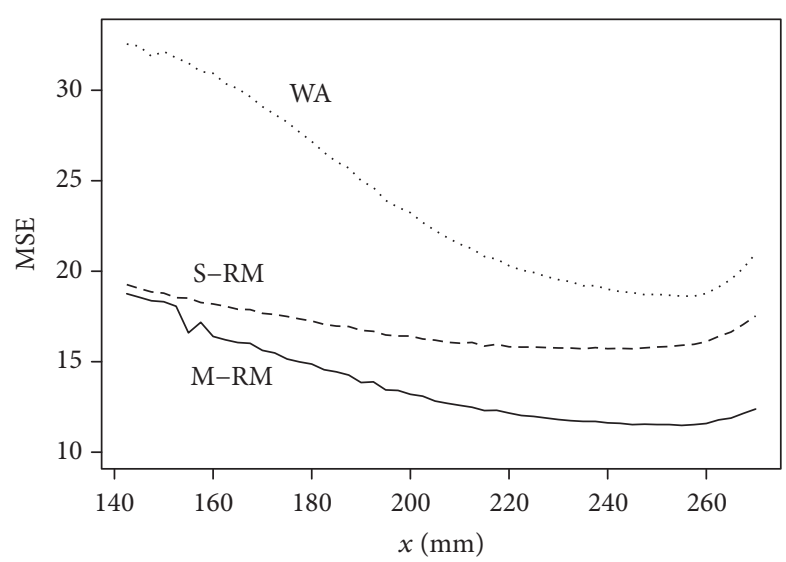

FIgURE 6: The MSE value for three predictive methods: WA (dotted line), S-RM (broken line) and M-RM (solid line). The horizontal axis indicates the distance $x(\mathrm{~mm})$ from the cutoff point. For M-RM, the MSE value indicates the value of $\operatorname{MSE}(105.0, x)$ with $x_{1}=105.0$ and $x_{2}=x$ in (10).

where $d_{i j}^{*}\left(x_{1}, x_{2}\right)$ is the predictive value of the diameter at the $j$ th point on the $i$ th yam with the location parameters $x_{1}$ and $x_{2}$.

A predictive model that minimizes the minimum values of $\operatorname{MSE}(x)$ and $\operatorname{MSE}\left(x_{1}, x_{2}\right)$ is considered to be the best model.

\section{Results}

First of all, as Step 0 of the proposed approach, measurements of diameter were disposed at equal intervals with $N=100$. For example, for the $i$-th yam with a length of $500 \mathrm{~mm}$ and a measuring interval of $50 \mathrm{~mm}$, we obtain the measurement of diameter as $\left\{y_{i, 10}, y_{i, 20}, \ldots, y_{i, 100}\right\}$, and $\left\{y_{i j}\right\}(j \neq 10,20, \ldots, 100)$ are missing. We then applied the Bayesian estimation model to estimate the diameters at every $j=1,2, \ldots, 100$ as Step 1 of the proposed approach. In Step 2, predictive models were constructed using the estimated values of parameters. In fact, three approaches of predicting yam shape, that is, WA, S-RM and M-RM, were applied to obtain the prediction for diameters. We set the position $x \mathrm{~mm}$ of the key diameter $D_{i}(x)$ to be $142.5,145.0, \ldots, 270.0$, and the MSE value was calculated for each value of $x$. In the case of M-RM, two positions $x_{1}$ and $x_{2}$ for defining the key diameters $D_{i}\left(x_{1}\right)$ and $D_{i}\left(x_{2}\right)$ were set as $\{85.0,87.5, \ldots, 142.0\}$ and $\{142.5,145.0, \ldots, 270.0\}$, respectively. The minimum MSE values of WA, S-RM, and M-RM were 18.62 (at $x=257.5 \mathrm{~mm}$ ), 15.71 (at $x=235.0 \mathrm{~mm}$ ), and 11.48 (at $x_{1}=105.0 \mathrm{~mm}$ and $x_{2}=255.0 \mathrm{~mm}$ ), respectively. Thus the minimum MSE value was attained by M-RM at $x_{1}=105.0 \mathrm{~mm}$ and $x_{2}=255.0 \mathrm{~mm}$. Figure 6 shows the change in the MSE value using the three methods. Figure 7 indicates the estimated coefficients $a_{j}, b_{j}$, and $c_{j}$ for $\mathrm{M}$ $\mathrm{RM}$ at $x_{1}=105.0 \mathrm{~mm}, x_{2}=255.0 \mathrm{~mm}$. The predictive value of the diameter $d_{j}^{*}$ at point $j$ is obtained by $d_{j}^{*}=$ $a_{j}+b_{j} D^{*}(105.0)+c_{j} D^{*}(255.0)$. We measure two diameters

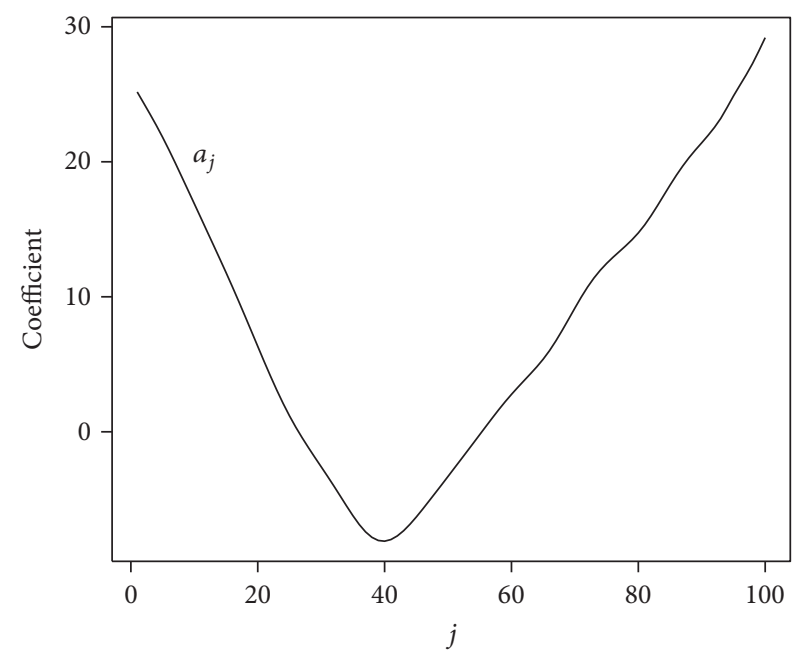

(a)

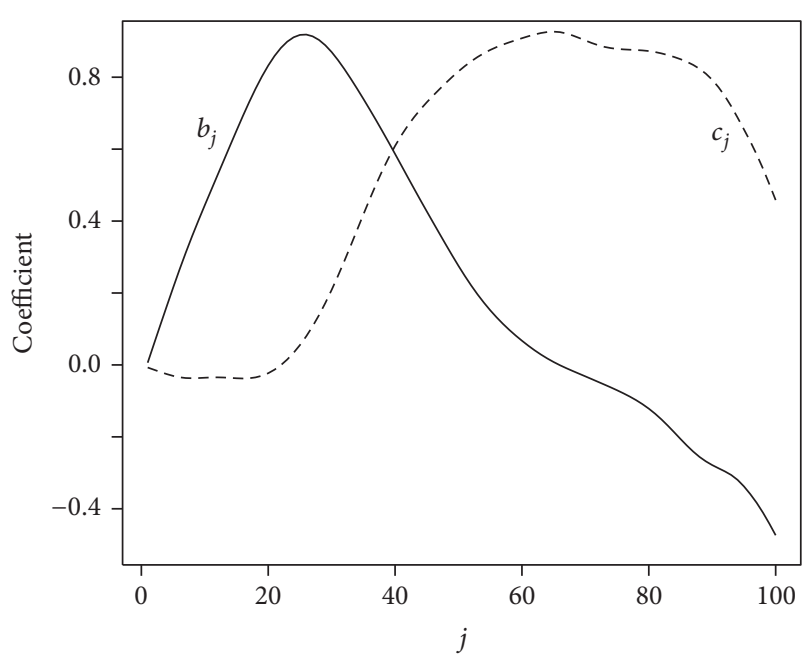

(b)

Figure 7: Estimates of multiple regression coefficients $a_{j}$ (a), $b_{j}$ (solid line in (b)), and $c_{j}$ (broken line in (b)). The predictive value of the diameter $d_{j}^{*}$ at point $j$ is obtained by $d_{j}^{*}=a_{j}+$ $b_{j} D^{*}(105.0)+c_{j} D^{*}(255.0)$. The diameter at $x=105.0 \mathrm{~mm}$ positively and negatively affected the prediction in the range of $j=1, \ldots, 50$ and $70, \ldots, 100$, respectively. Another diameter at $x=255.0 \mathrm{~mm}$ contributed to the estimate for the range of $j=50, \ldots, 90$.

$D^{*}(105.0)$ and $D^{*}(255.0)$ of a new yam for whole shape prediction. Figures 8 and 9 show observations together with predictions of the diameters at each point using M-RM with two key diameters at $x=105.0$ and $255.0 \mathrm{~mm}$ for the shape of the 111 samples in this study.

\section{Discussion}

First, three predictive models, WA, S-RM, and M-RM, which are constructed based on result of the Bayesian estimation model, for yam shape prediction are compared in terms of MSE. Although WA is a simple approach compared with the other methods, it resulted in a small MSE value of 18.62 at $x=$ $257.5 \mathrm{~mm}$. The regression methods performed better than 

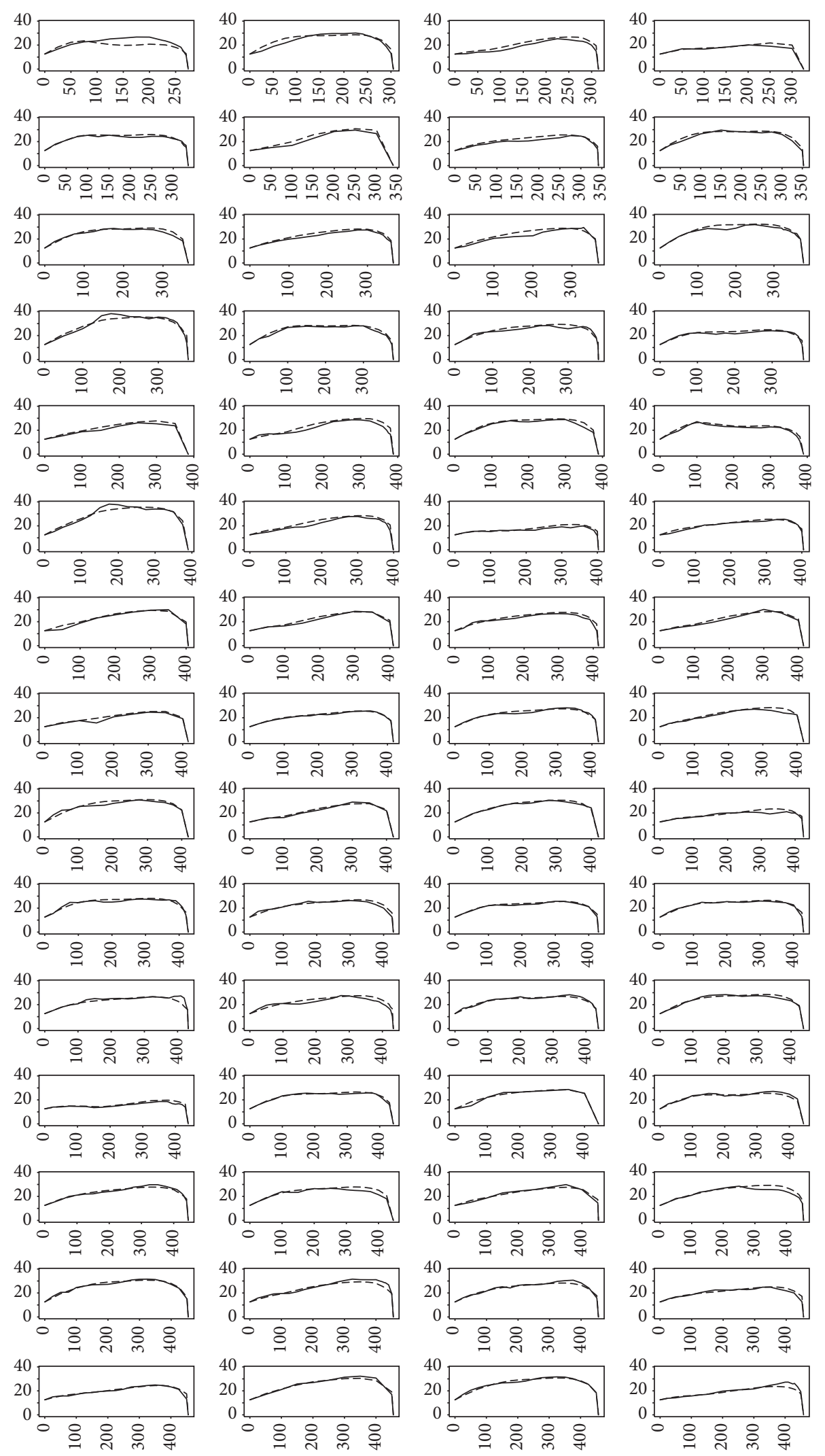

FIGURE 8: Observations (solid) and predictions (broken) for the shape of the samples (numbers 1-60, length of 274 to $459 \mathrm{~mm}$ ), using proposed Bayesian method with M-RM prediction model. The samples are ordered by the length. The horizontal and vertical axes indicate the distance $(\mathrm{mm})$ from the cutoff point and the radius $(\mathrm{mm})$, respectively. 

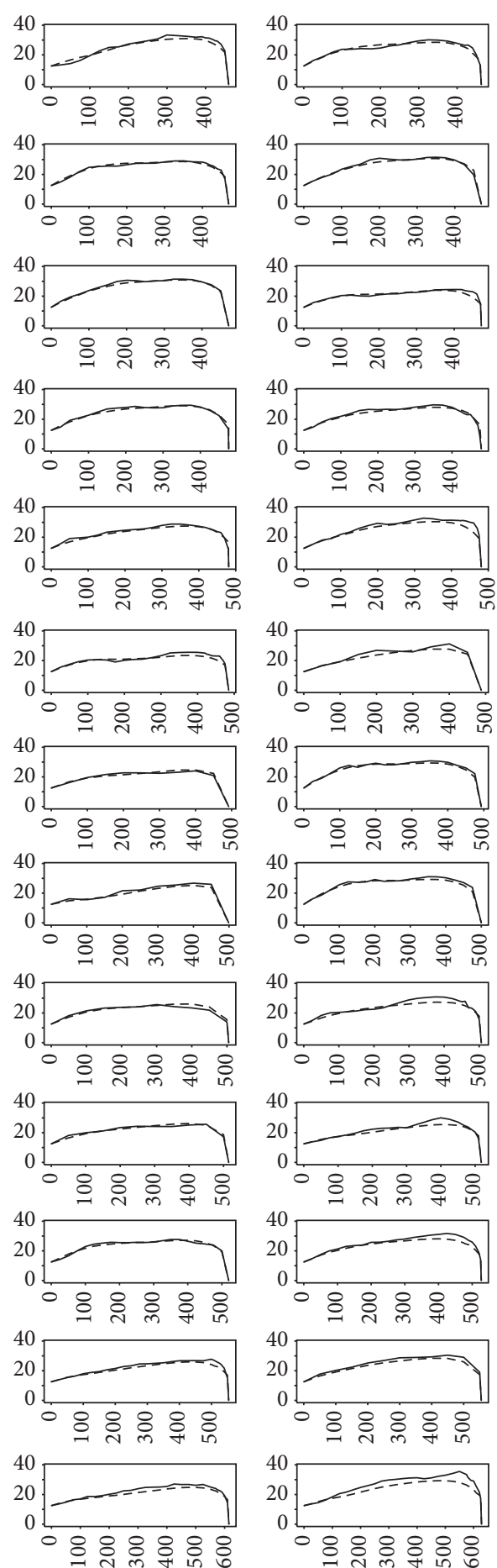
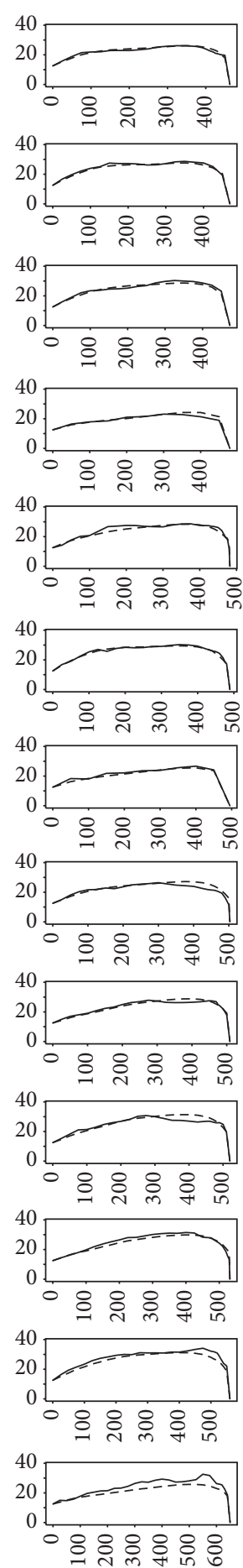
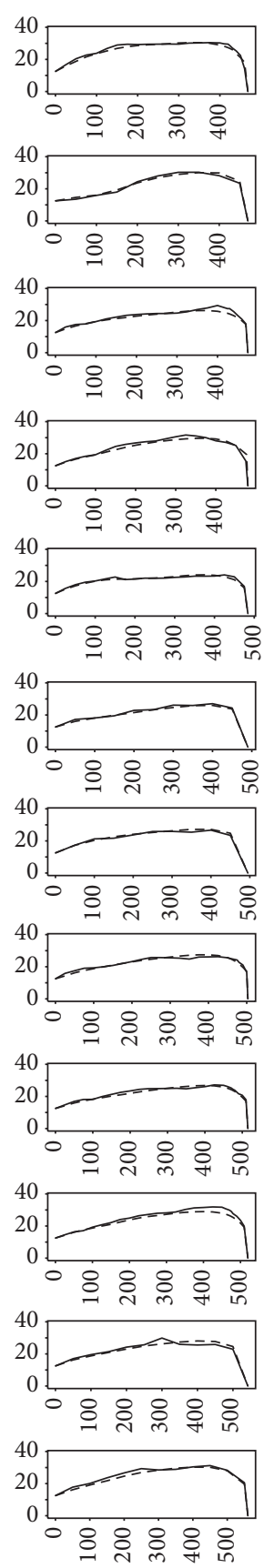

FIGURE 9: Observations (solid) and predictions (broken) for the shape of the samples (numbers 61-111, length of 460 to $650 \mathrm{~mm}$ ), using proposed Bayesian method with M-RM prediction model. The samples are ordered by the length. The horizontal and vertical axes indicate the distance $(\mathrm{mm})$ from the cutoff point and the radius $(\mathrm{mm})$, respectively.

WA; the MSE was 15.71 for S-RM at $x=235.0 \mathrm{~mm}$ and 11.48 for M-RM at $x_{1}=255.0 \mathrm{~mm}$ and $x_{2}=105.0 \mathrm{~mm}$. According to Figure 7 for the coefficients in M-RM, the diameter at $x=$ $105.0 \mathrm{~mm}$ positively and negatively affected the prediction in the range of $j=1, \ldots, 50$ and $70, \ldots, 100$, respectively. Another diameter at $x=255.0 \mathrm{~mm}$ contributed to the estimate for the range of $j=50, \ldots, 90$. The two diameters can improve the performance of the estimate through the two coefficients.

After the construction of M-RM using the sample data set in this study, M-RM can be used for whole shape prediction based on two diameters at fixed positions of $x=105.0$ and 


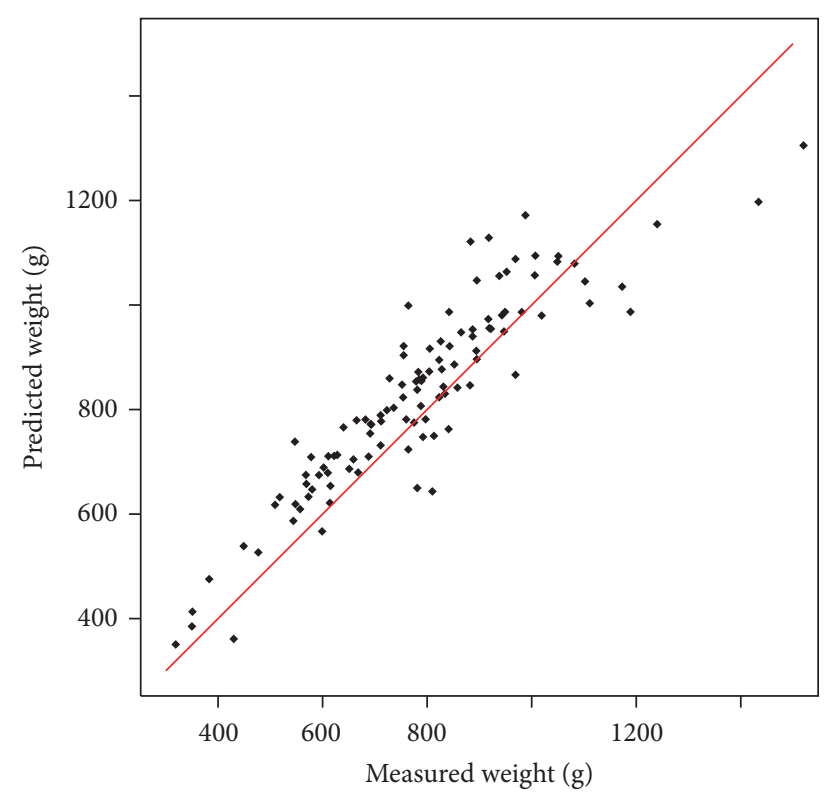

FIGURE 10: Measured and predicted weight by using proposed Bayesian method with M-RM prediction model. The proposed Bayesian method successfully predicted not only the whole shape of yams (Figures 8 and 9) but also the weight of the yams.

$255.0 \mathrm{~mm}$. The quality of the sample data set is then critical for the performance of the shape prediction. In our data set, yam length and weight were correlated with each other $(r=0.739, p<0.001$, Appendix A). This means that the yams had a uniform shape and there were no outliers that show an irregular shape; if there were thick (short and heavy) and thin (long and light) yams, they might be plotted on the upper-left or lower-right on the scatterplot respectively, and the correlation might be lower. The quality of the sample data set, which was used for the construction of M-RM, seemed to be high for model construction.

The M-RM method performed well according to the MSE value (Figure 6) and visual inspection of the actual shape prediction (Figures 8 and 9). In order to evaluate the weight of the yams based on the predicted shape, we assumed that (a) each yam was circular in cross-section and (b) the shape changed linearly between each pair of positions. The weight was then estimated under the assumption (a) and (b) (Figure 10). M-RM successfully predicted the weight of the yams. Relatively high accuracy can be obtained by adequately treating the outliers (e.g., removing heavy yams with weight $>1200 \mathrm{~g}=$ mean $+2 \mathrm{SD}$ ). We believe that the Bayesian approaches in this paper are applicable not only for shape prediction of yam but also for other shape prediction problems in agriculture.

\section{Conclusion}

This paper proposed Bayesian methods, which is a combination of Bayesian estimation model and predictive model, for shape prediction of yam. Three predictive models we applied were weighted average (WA) and single and multiple

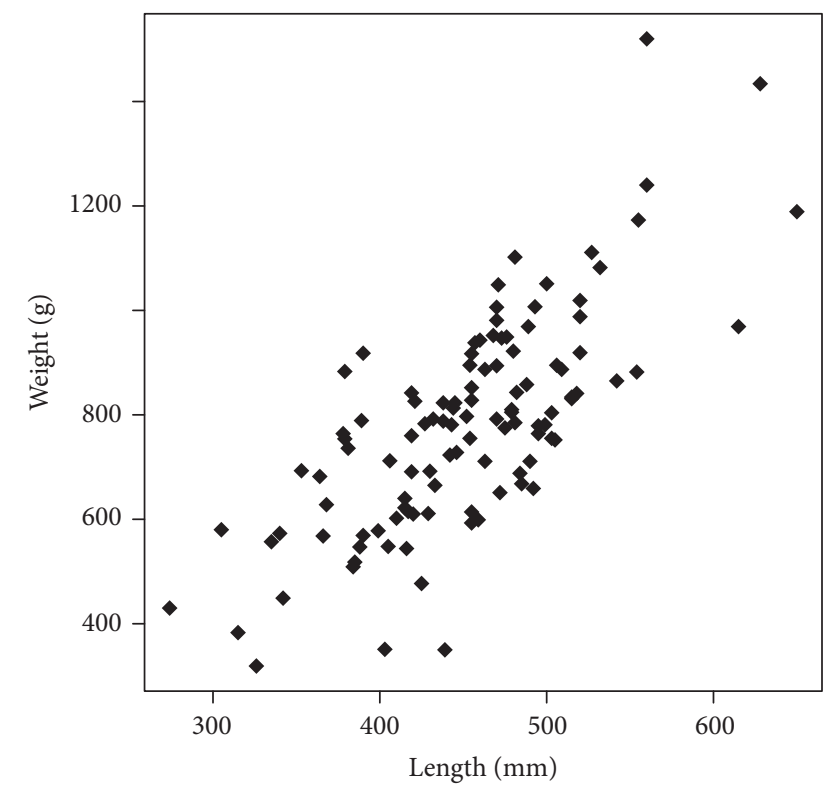

FIGURE 11: The scatter plot of the length and weight of the 111 yams in this study.

regression methods (S-RM and $\mathrm{M}-\mathrm{RM}$, resp.). Bayesian method with M-RM prediction model with two diameters at fixed positions of $x=105.0$ and $255.0 \mathrm{~mm}$ attained the highest performance of the estimate in terms of the MSE value. After the construction of M-RM using the sample data set in this study, M-RM predicts the whole shape of yam based on two key diameters. To measure two diameters at those positions of a yam is fairly easy, and this approach does not need any sensors for the shape estimation. Development of such shape prediction approaches, including our Bayesian method, will be required to reduce the cost and time in food processing.

\section{Appendix}

\section{A. Detailed Data for the Sample Yam Data}

Figure 11 shows the scatterplot of the length and weight of the 111 yams in this study. Length and weight were highly correlated with each other (Pearson correlation coefficient $r=0.739, p<0.001)$, implying high quality of the data for model construction.

\section{B. Algorithm for Estimating the Diameters}

For a given sample $i$, let $\mathbf{z}_{i 0}$ denote the initial value of the state and let $Y_{i m}=\left\{y_{i n} ; n=1,2, \ldots, m\right\}$ denote a set of observations up to the time point $m$ for the $i$-th sample. Assume that $\mathbf{z}_{i 0} \sim N\left(\mathbf{z}_{i|| 0}, \mathbf{C}_{i|0| 0}\right)$. It is well-known that the distribution $f\left(\mathbf{z}_{i j} \mid Y_{i m}\right)$ for the state $\mathbf{z}_{i j}$ conditionally on $Y_{i m}$ is Gaussian, so it is only necessary to obtain the mean $\mathbf{z}_{i j \mid m}$ and the covariance matrix $\mathbf{C}_{i j \mid m}$ of $\mathbf{z}_{i j}$ with respect to $f\left(\mathbf{z}_{i j} \mid Y_{m}\right)$.

When the values of $\sigma^{2}$ and $\tau_{i}^{2}$, the initial distribution $N\left(\mathbf{z}_{i 0 \mid 0}, \mathbf{C}_{i 0 \mid 0}\right)$, and an observation set $Y_{i N}$ up to the point $N$ 
are given, then the estimates for the state $\mathbf{z}_{i j}$ can be obtained using the well-known Kalman filter (for $j=1,2, \ldots, N$ ) and the fixed-interval smoothing (for $j=N-1, N-2, \ldots, 1$ ) recursively as follows (see, e.g., $[18,20])$.

Kalman Filter (Step 1): One-Step Ahead Prediction

$$
\begin{aligned}
\mathbf{z}_{i j \mid j-1} & =\mathbf{F} \mathbf{z}_{i(j-1) \mid j-1}, \\
\mathbf{C}_{i j \mid j-1} & =\mathbf{F C}_{i(j-1) \mid j-1} \mathbf{F}^{\mathrm{t}}+\tau_{i}^{2} \mathbf{G G}^{\mathrm{t}} .
\end{aligned}
$$

Kalman Filter (Step 2): Filter

$$
\begin{aligned}
\mathbf{L}_{i j} & =\mathbf{C}_{i j \mid j-1} \mathbf{H}^{\mathrm{t}}\left(\mathbf{H C}_{i j \mid j-1} \mathbf{H}^{\mathrm{t}}+\sigma^{2}\right)^{-1}, \\
\mathbf{z}_{i j \mid j} & =\mathbf{z}_{i j \mid j-1}+\mathbf{L}_{i j}\left(y_{i j}-\mathbf{H} \mathbf{z}_{i j \mid j-1}\right), \\
\mathbf{C}_{i j \mid j} & =\left(\mathbf{I}-\mathbf{L}_{i j} \mathbf{H}\right) \mathbf{C}_{i j \mid j-1} .
\end{aligned}
$$

Fixed-Interval Smoothing

$$
\begin{aligned}
\mathbf{P}_{i j} & =\mathbf{C}_{i j \mid j} \mathbf{F}^{\mathrm{t}} \mathbf{C}_{i(j+1) \mid j}^{-1}, \\
\mathbf{z}_{i j \mid N} & =\mathbf{z}_{i j \mid j}+\mathbf{P}_{i j}\left(\mathbf{z}_{i(j+1) \mid N}-\mathbf{z}_{i(j+1) \mid n}\right), \\
\mathbf{C}_{i j \mid N} & =\mathbf{C}_{i j \mid j}+\mathbf{P}_{i j}\left(\mathbf{C}_{i(j+1) \mid N}-\mathbf{C}_{i(j+1) \mid j}\right) \mathbf{P}_{i j}^{\mathrm{t}} .
\end{aligned}
$$

Here, I denotes an identity matrix. Note that the calculation in the filter step will be skipped when $y_{i j}$ is a missing value.

Then, the posterior distribution of $\mathbf{z}_{i j}$ can be given by $\mathbf{z}_{i j \mid N}$ and $\mathbf{C}_{i j \mid N}$, and subsequently the estimates for the parameter $d_{i j}$ can be obtained because the state space model described by (4) in the main text incorporates $d_{i j}$ in the state vector $\mathbf{z}_{i j}$. Hereafter, the estimates of $d_{i j}$ are denoted by $\widehat{d}_{i j}$.

\section{Algorithm for Estimating the Variances}

When the observation data $Y_{i N}=\left\{y_{i 1}, y_{i 2}, \ldots, y_{i N}\right\}$ for the $i$-th sample are given, a likelihood function for the variances $\sigma^{2}$ and $\tau_{i}^{2}$ is defined approximately by

$$
f\left(Y_{i N} \mid \sigma^{2}, \tau_{i}^{2}\right)=\prod_{m=1}^{N} f_{m}\left(y_{i m} \mid Y_{i(m-1)} ; \sigma^{2}, \tau_{i}^{2}\right),
$$

where $f_{m}\left(y_{i m} \mid Y_{i(m-1)} ; \sigma^{2}, \tau_{i}^{2}\right)$ is the conditional density function of $y_{i m}$ given the past history $Y_{i(m-1)}=\left\{y_{i(m-1)}\right.$, $\left.y_{i(m-2)}, \ldots\right\}$. Assume that $Y_{i 0}=\left\{y_{i 0}, y_{i(-1)}, \ldots\right\}$ is an empty set, then $f_{1}\left(y_{i 1} \mid Y_{i 0} ; \sigma^{2}, \tau_{i}^{2}\right)=f_{1}\left(y_{i 1} \mid \sigma^{2}, \tau_{i}^{2}\right)$. By taking the logarithm of $f\left(Y_{i N} \mid \sigma^{2}, \tau_{i}^{2}\right)$, the log-likelihood is obtained as

$$
\begin{aligned}
\ell\left(\sigma^{2}, \tau_{i}^{2}\right) & =\log f\left(Y_{i N} \mid \sigma^{2}, \tau_{i}^{2}\right) \\
& =\sum_{m=1}^{N} \log f_{m}\left(y_{i m} \mid Y_{i(m-1)} ; \sigma^{2}, \tau_{i}^{2}\right) .
\end{aligned}
$$

As given by [18] under the use of the Kalman filter, the conditional density $f_{m}\left(y_{i m} \mid Y_{i(m-1)} ; \sigma^{2}, \tau_{i}^{2}\right)$ is a normal density given by

$$
\begin{aligned}
f_{m} & \left(y_{i m} \mid Y_{i(m-1)} ; \sigma^{2}, \tau_{i}^{2}\right) \\
= & \frac{1}{\sqrt{2 \pi w_{i m \mid m-1}}} \exp \left\{-\frac{\left(y_{i m}-\widehat{y}_{i m \mid m-1}\right)^{2}}{2 w_{i m \mid m-1}}\right\},
\end{aligned}
$$

where $\widehat{y}_{i m \mid m-1}$ is the one-step ahead prediction for $y_{i m}$ and $w_{i m \mid m-1}$ is the variance of the predictive error, given by

$$
\begin{aligned}
\widehat{y}_{i m \mid m-1} & =\mathbf{H z}_{i m \mid m-1}, \\
w_{i m \mid m-1} & =\mathbf{H C}_{i m \mid m-1} \mathbf{H}^{\mathrm{t}}+\sigma^{2},
\end{aligned}
$$

respectively.

Thus, the estimates of $\sigma^{2}$ and $\tau_{i}^{2}$ can be obtained using the maximum likelihood method. Specifically, for a given value of $\sigma^{2}$, we can obtain the estimate $\widehat{\tau}_{i}^{2}$ of $\tau_{i}^{2}$ for $i=1,2, \ldots$ by maximizing $\ell\left(\sigma^{2}, \tau_{i}^{2}\right)$ in (C.2) numerically. Then, the estimate $\widehat{\sigma}^{2}$ for $\sigma^{2}$ is obtained similarly by maximizing

$$
\bar{\ell}\left(\sigma^{2}\right)=\frac{1}{M} \sum_{i=1}^{M} \ell\left(\sigma^{2}, \widehat{\tau}_{i}^{2}\right) \text {. }
$$

By applying the results of $\widehat{\sigma}^{2}$ and $\widehat{\tau}_{i}^{2}$ to the above algorithms of the Kalman filter and fixed-interval smoothing, we can obtain the final estimates of $d_{i j}$ and corresponding variances from the results of $\mathbf{z}_{i j \mid N}$ and $\mathbf{C}_{i j \mid N}$.

\section{Conflicts of Interest}

The authors declare that there are no conflicts of interest regarding the publication of this paper.

\section{References}

[1] Trade Statistics of Japan. http://www.customs.go.jp/toukei/info/ index_e.htm.

[2] Portal Site of Official Statistics in Japan. http://www.e-stat.go.jp/ SG1/estat/eStatTopPortalE.do.

[3] G. P. Moreda, J. Ortiz-Cañavate, F. J. García-Ramos, and M. Ruiz-Altisent, "Non-destructive technologies for fruit and vegetable size determination-a review," Journal of Food Engineering, vol. 92, no. 2, pp. 119-136, 2009.

[4] N. Kondo, "Automation on fruit and vegetable grading system and food traceability," Trends in Food Science \& Technology, vol. 21, pp. 145-152, 2010.

[5] X. Liming and Z. Yanchao, "Automated strawberry grading system based on image processing," Computers and Electronics in Agriculture, vol. 71, no. 1, pp. S32-S39, 2010.

[6] C. Costa, F. Antonucci, F. Pallottino, J. Aguzzi, D. Sun, and P. Menesatti, "Shape analysis of agricultural products: a review of recent research advances and potential application to computer vision," Food and Bioprocess Technology, vol. 4, no. 5, pp. 673692, 2011. 
[7] S. Goto, H. Iwata, S. Shibano, K. Ohya, A. Suzuki, and H. Ogawa, "Fruit shape variation in Fraxinus mandshurica var. japonica characterized using elliptic Fourier descriptors and the effect on flight duration," Ecological Research, vol. 20, no. 6, pp. 733-738, 2005.

[8] M. Z. Abdullah, J. Mohamad-Saleh, A. S. Fathinul-Syahir, and B. M. N. Mohd-Azemi, "Discrimination and classification of fresh-cut starfruits (Averrhoa carambola L.) using automated machine vision system," Journal of Food Engineering, vol. 76, no. 4, pp. 506-523, 2006.

[9] S. Borah, E. L. Hines, and M. Bhuyan, "Wavelet transform based image texture analysis for size estimation applied to the sorting of tea granules," Journal of Food Engineering, vol. 79, no. 2, pp. 629-639, 2007.

[10] K. Moon Sung, C. Yud-Ren, C. Byoung-Kwan et al., "Hyperspectral reflectance and fluorescence line-scan imaging for online defect and fecal contamination inspection of apples," Sensing and Instrumentation for Food Quality and Safety, vol. 1, pp. 151-159, 2007.

[11] H. Sadrnia, A. Rajabipour, A. Jafary, J. Arzhang, and Y. Mostofi, "Classification and analysis of fruit shapes in long type watermelon using image processing," International Journal of Agricultural and Biological Engineering, vol. 1, pp. 68-70, 2007.

[12] P. Menesatti, C. Costa, G. Paglia et al., "Shape-based methodology for multivariate discrimination among Italian hazelnut cultivars," Biosystems Engineering, vol. 101, no. 4, pp. 417-424, 2008.

[13] C. Costa, P. Menesatti, G. Paglia et al., "Quantitative evaluation of Tarocco sweet orange fruit shape using optoelectronic elliptic Fourier based analysis," Postharvest Biology and Technology, vol. 54, no. 1, pp. 38-47, 2009.

[14] C. B. Singh, R. Choudhary, D. S. Jayas, and J. Paliwal, "Wavelet analysis of signals in agriculture and food quality inspection," Food and Bioprocess Technology, vol. 3, no. 1, pp. 2-12, 2010.

[15] A. Mizushima and R. Lu, "An image segmentation method for apple sorting and grading using support vector machine and Otsu's method," Computers and Electronics in Agriculture, vol. 94, pp. 29-37, 2013.

[16] M.-H. Hu, Q.-L. Dong, B.-L. Liu, and P. K. Malakar, "The potential of double k-means clustering for banana image segmentation," Journal of Food Process Engineering, vol. 37, no. 1, pp. 10-18, 2014.

[17] A. Gelman, B. John Carlin, S. Hal Stern, B. David Dunson, A. Vehtari, and B. Donald Rubin, Bayesian Data Analysis, vol. 2, CRC Press, Boca Raton, FL, USA, 2014.

[18] G. Kitagawa and W. Gersch, Smoothness Priors Analysis of Time Series, vol. 116, Springer, 1996.

[19] K. Koki and M. Hachiya, "A statistical approach of identifying indexes crucial to characterizing chinese yams in terms of shape," in Advances in Intelligent Systems, Ford Lumban Gaol, Zenon Chaczko, Kiyota Hashimoto, T. Matsuo and W. Grosky, Eds., pp. 27-34, WIT Press, 2014.

[20] D. O. Brian Anderson and B. John Moore, Optimal Filtering, Prentice-Hall, Inc, 1979.

[21] D. Hrishikesh, V. Ullah, and A. Ullah, Recent Advances in Regression Methods, Marcel Dekker, Inc, 1981. 


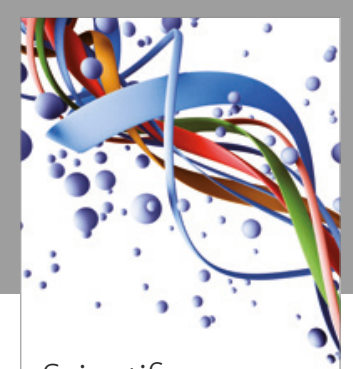

Scientifica
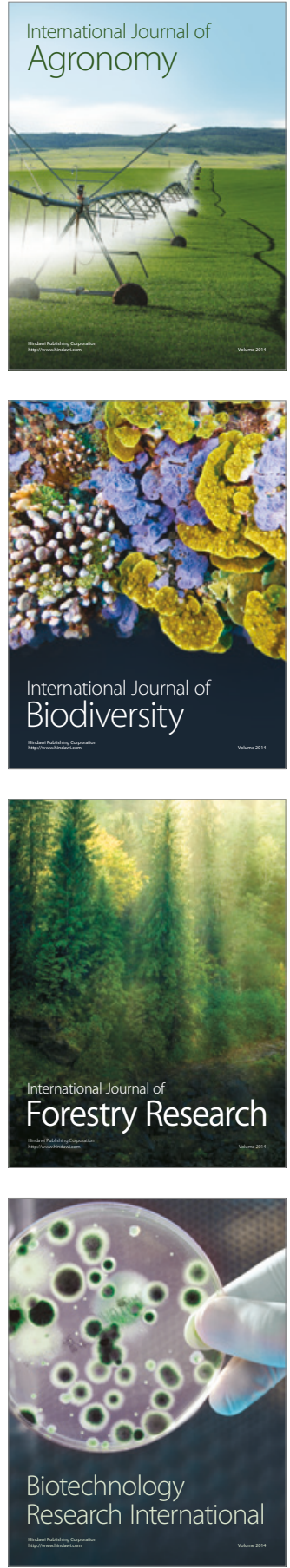
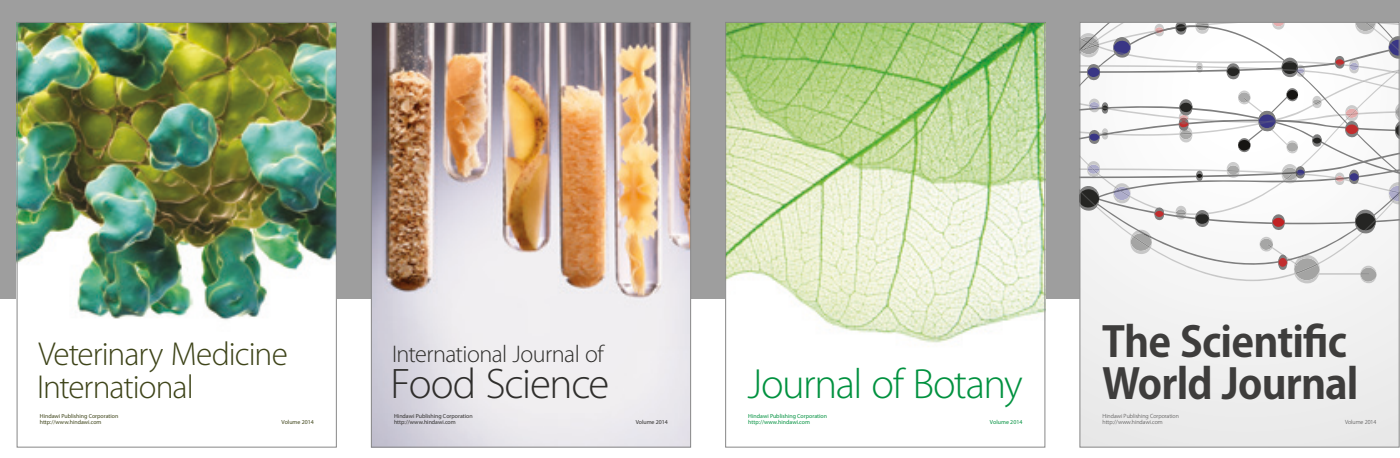

The Scientific

\section{World Journal}

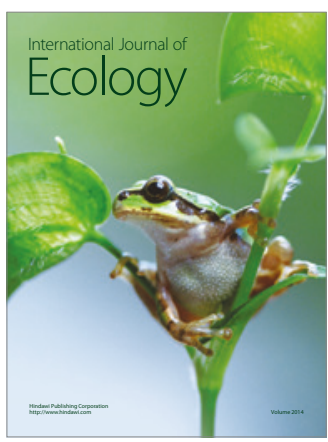

\section{Hindawi}

Submit your manuscripts at

https://www.hindawi.com
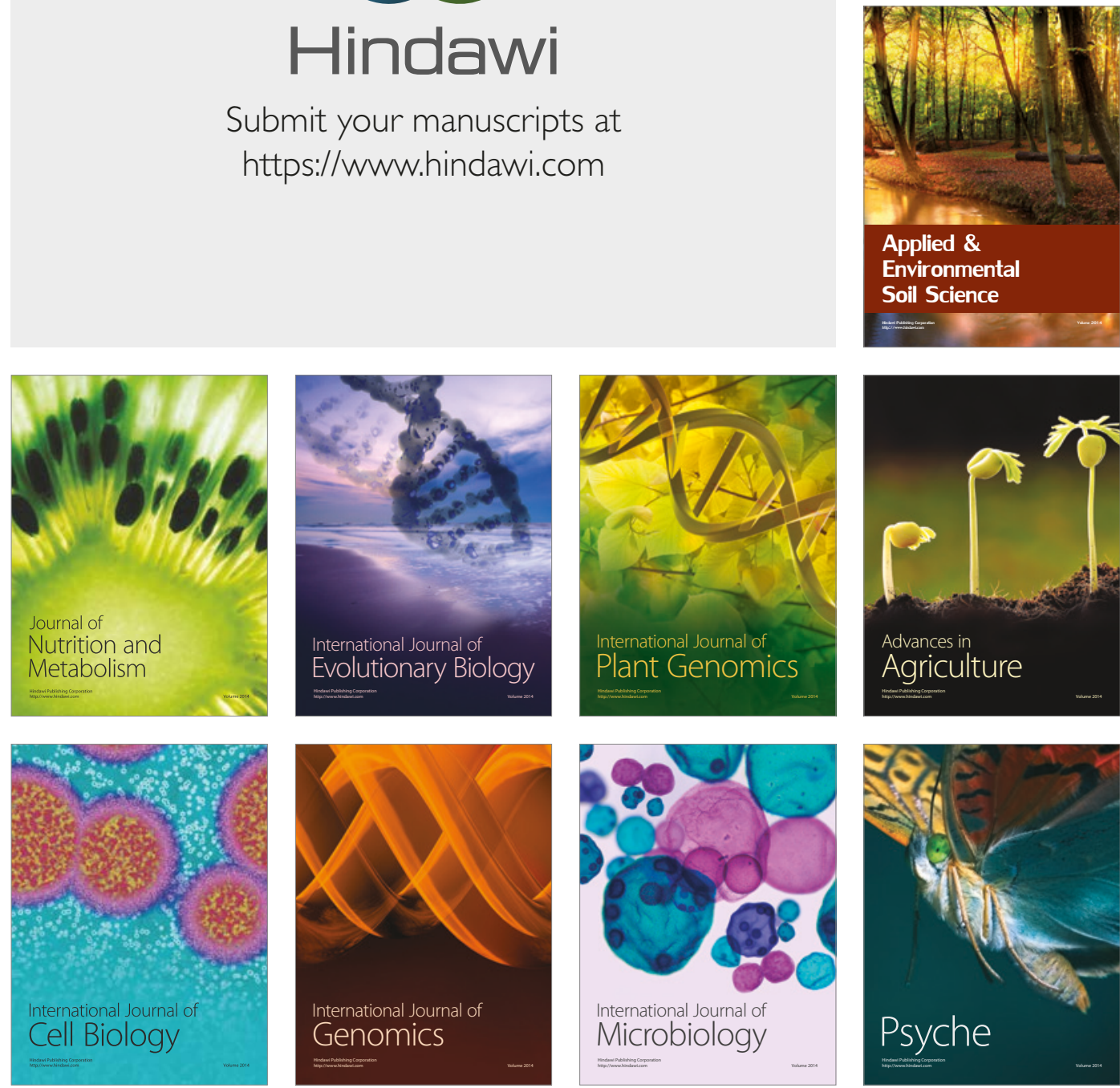

hternational Journal of Microbiology
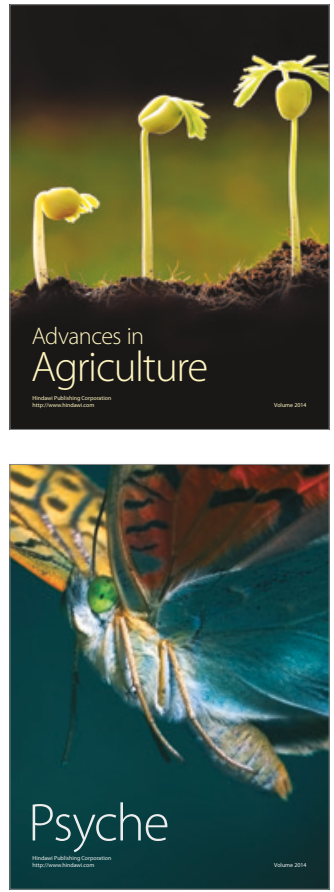